\title{
Evaluación del efecto acaricida de Momordica charantia, Megaskepasma erythrochlamys y Gliricidia sepium sobre Rhipicephalus microplus
}

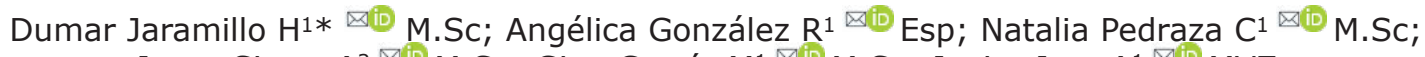 \\ Jorge Sierra $A^{2} \bowtie \mathbb{B}$ M.Sc; Gina García M1 ${ }^{\otimes \mathbb{E}}$ M.Sc; Javier Jara A ${ }^{\circledR \mathbb{E}}$ MVZ.
}

\begin{abstract}
${ }^{1}$ Universidad de los Llanos, Facultad de Ciencias Agropecuarias y Recursos Naturales, Escuela de Ciencias Animales, Research group in Experimental Pharmacology and internal medicine -ÉLITE, Km 12 Vía Puerto López Villavicencio, Colombia.

2Universidad de los Llanos, Facultad de Ciencias Básicas e Ingeniería, Departamento de Química. Research group in Experimental Pharmacology and Internal Medicine -ÉLITE.

*Correspondencia: dumar.jaramillo@unillanos.edu.co
\end{abstract}

Recibido: Febrero 2018; Aceptado: Agosto 2019; Publicado: Diciembre 2019.

\section{RESUMEN}

Objetivo. Se evaluó la actividad acaricida de Momordica charantia (Mc), Megaskepasma erythrochlamys (Me) y Gliricidia sepium (Gs) sobre Rhipicephalus microplus (Rm). Materiales y métodos. Se realizó la marcha fitoquímica preliminar de hojas del extracto metanólico de Mc (EMc), del extracto etanólico de $M e(E M e)$ y del extracto acetónico de Gs (EGs) a través de la técnica de colorimetría y cromatografía en capa delgada (CCD). La actividad acaricida se realizó a través de pruebas in-vitro utilizando la prueba de inmersión de larvas (LIT) y la prueba de inmersión de adultos (AIT). Para las pruebas in-situ se usaron bovinos en pastoreo infestados naturalmente con garrapatas, utilizando las $\mathrm{CL}_{50}$ obtenidas en las pruebas in-vitro AIT; posteriormente las teleoginas se llevaron a incubación para evaluar su capacidad reproductiva. Resultados. Se determinó la presencia de varios grupos de metabolitos secundarios de interés acaricida. Se demostró el efecto acaricida de los extractos de las plantas sobre teleoginas; aunque sólo EGs mostró actividad larvicida. Los extractos a $160 \mathrm{mg} / \mathrm{mL}$ afectaron el ciclo de vida de Rm inhibiendo la ovoposición en un 46.9\%, $66.1 \%$ y $84.03 \%(p<0.05)$ para $E G s, E M c$ y $E M e$, respectivamente. Por otro lado, en las pruebas in situ se observó diferencia significativa $(p<0.05)$ entre el tratamiento de EMc y EMe respecto a los grupos controles. Conclusiones. Los resultados obtenidos son prometedores para fortalecer la posibilidad de vinculación de los extractos de estas plantas dentro de planes integrados de control de garrapatas en sistemas de producción de bovinos.

Palabras clave: ácaros y garrapatas, enfermedades de los bovinos, etnofarmacología (Fuente: DeSC).

\section{ABSTRACT}

Objective. The acaricidal activity of Momordica charantia (Mc), Megaskepasma erythrochlamys (Me) and Gliricidia sepium (Gs) on Rhipicephalus microplus (Rm) was evaluated. Materials and methods. Preliminary phytochemical analysis of leaves of the methanolic extract of Mc (EMc), the ethanolic extract of $M e(E M e)$ and the acetone extract of Gs (EGs) were carried out through the technique of colorimetry and thin layer chromatography (CCD). The acaricidal activity was performed through in-vitro tests using the larval immersion test (LIT) and the adult immersion test (AIT). For in-situ tests, grazing cattle naturally infested with ticks were used, using the $\mathrm{LC}_{50}$ obtained from the in-vitro AIT tests; later the teleogines were taken to incubation to evaluate their reproductive capacity. Results. The presence of several groups of secondary metabolites of Como citar (Vancouver).

Jaramillo HD, González RA, Pedraza CN, Sierra AG, García MG, Jara AR. Efecto acaricida de Momordica charantia, Megaskepasma erythrochlamys y Gliricidia sepium sobre Rhipicephalus microplus. Rev MVZ Cordoba. 2020; 25(1):e1951. DOI: https://doi.org/10.21897/rmvz.1951 
acaricidal interest was determined. The acaricidal effect of the extracts of the plants on teleogines was demonstrated; although only EGs showed larvicidal activity. Extracts at $160 \mathrm{mg} / \mathrm{mL}$ affected the life cycle of Rm by inhibiting ovoposition in $46.9 \%, 66.1 \%$ and $84.03 \%(p<0.05)$ for EGs, EMc and EMe, respectively. On the other hand, the in-situ tests showed a significant difference $(p<0.05)$ between the treatment of EMc and EMe with respect to the control groups. Conclusions. The results obtained are promising to strengthen the possibility of linking the extracts of these plants into integrated plans for the control of ticks in cattle systems.

Keywords: Acari, cattle diseases, ethnopharmacology (Source: DeSC).

\section{INTRODUCCIÓN}

La ganadería bovina es una actividad sobresaliente en el contexto económico nacional y genera el $3.5 \%$ del PIB nacional y el $56 \%$ del PIB pecuario (1). Los ectoparásitos, específicamente la garrapata $\mathrm{Rm}$ ha sido asociada con grandes pérdidas económicas para este reglón productivo por la disminución de los índices reproductivos y la ganancia de peso, asociados a la transmisión de agentes patógenos (2). El uso de acaricidas ha tenido una eficacia limitada en la reducción de las infestaciones y es acompañada de problemas de cepas de garrapatas resistentes, contaminación ambiental y de productos cárnicos o lácteos $(3,4)$; esto refuerza la necesidad de enfoques alternativos para controlar las infestaciones de garrapatas, donde los extractos vegetales son una opción dentro de la incursión del manejo integral de parasitosis externa $(5,6)$.

Los productores quienes a través de conocimientos tradicionales existentes en la flora han buscado y encontrado soluciones para el control de ectoparásitos; especies como matarratón (Gs) y Melón amargo (Mc) fueron reportados con efecto antiparasitario en Colombia (7).

El objetivo del presente trabajo de investigación fue validar la información etnofarmacológica del uso de estas plantas forrajeras, además de manto rojo $(M e)$, como antiparasitario para el control de $\mathrm{Rm}$, tanto en modelos in vitro como in situ.

\section{MATERIALES Y MÉTODOS.}

Colecta de plantas y elaboración de extractos. Hojas de MC L. N ${ }^{\circ} \mathrm{COL} 580477, \mathrm{Me}$ Lindau $\mathrm{N}^{\circ} \mathrm{COL} 575462$ y Gs (Jacq.) Kunth ex Walp N ${ }^{\circ} \mathrm{COL576461}$; se colectaron entre los meses de abril y julio, en la granja de la Universidad de los Llanos, ubicada en la vereda Barcelona, Villavicencio (Meta). $4^{\circ} 04^{\prime} 32,91^{\prime \prime}$ Latitud Norte, $73^{\circ} 35^{\prime} 02,27$ Latitud Oeste, altitud 386 msnm, precipitación de 3500 mm/año, HR 87\% y temperatura ambiental promedio $27^{\circ} \mathrm{C}$.
El material vegetal se trató de acuerdo a la metodología de análisis fitoquímico propuesta por Sanabria, 1983 (8), así: fue secado en un horno de aire circulante a $40^{\circ} \mathrm{C}$ durante $72 \mathrm{~h}$ y posteriormente molido hasta pulverización. Para la elaboración de los extractos se pesaron $150 \mathrm{~g}$ de material vegetal seco molido de Mc, Me y Gs; posteriormente expuestos a $600 \mathrm{~mL}$ de Metanol 99.8\%, Etanol $99.8 \%$ y Acetona $70 \%$ (SigmaAldrich, St. Louis, MO), respectivamente; cada uno en un embudo de decantación de vidrio, durante 72 horas, llevando a cabo la técnica de percolación continua hasta agotamiento.

El líquido percolado se filtró y concentró a $40^{\circ} \mathrm{C}$ en un evaporador rotatorio IKA ${ }^{\circledR}$ RV10 control, a presión reducida, llevándose a sequedad finalmente en baño de María. Los extractos fueron transferidos a viales de vidrio ámbar y conservados a $4^{\circ} \mathrm{C}$ hasta el momento de realizar las pruebas, el rendimiento promedio del proceso de extracción fue del $5 \%$, posteriormente se realizó análisis fitoquímico preliminar de los extractos con el fin de determinar la presencia de metabolitos secundarios a través de la técnica de colorimetría y CCD.

Colonia de garrapatas. La colecta de teleoginas se desarrolló en el hato bovino (21 animales parasitados naturalmente) de la granja de la Universidad de los Llanos; las teleoginas se lavaron con una solución de hipoclorito $2.5 \%$ (6); grupos de 10 teleoginas se colocaron sobre papel kraft en cajas de Petri que luego fueron ubicadas en cajas plásticas para la generación de microambientes e incubadas utilizando una incubadora refrigerada MEMMERT ${ }^{\circledR}$ IPP 110 a $27-28^{\circ} \mathrm{C}$ y HR $85-95 \%$.

Catorce días posteriores a la incubación, los huevos de los grupos de teleoginas son pesados, identificados y colocados en un vial de vidrio individual; los viales son tapados con un tapón de gasa y posteriormente colocados en las cajas plásticas para su incubación; una vez eclosionados, las larvas fueron utilizadas en las pruebas acaricidas in vitro.

Pruebas in vitro. Se aplicó la prueba AIT propuesta por Drummond et al (9), 
con adaptaciones. Las teleoginas fueron seleccionadas de acuerdo a su morfología, motilidad y máximo de ingurgitamiento; luego pesadas y separadas en grupos de 10 con pesos homogéneos. Seis repeticiones para cada concentración incluyendo el grupo control negativo (agua-Tween $802.5 \%$ ) y el control positivo Amitraz $0.025 \%$ se desarrollaron, donde las teleoginas se expusieron a los extractos en concentraciones decrecientes de 320, 160, $80,40,20$ y $10 \mathrm{mg} / \mathrm{mL}$ vehiculizados en aguaTween 80 2.5\%; sumergiéndolas en $10 \mathrm{~mL}$ de estas soluciones durante 10 minutos, después secadas y colocadas en cajas de Petri plásticas, luego incubadas en las mismas condiciones descritas con anterioridad.

Mediante estereoscopia se determinó la mortalidad a las 24 horas, 7 días y 14 días postratamiento; el día 14 postinmersión los huevos fueron colectados y pesados, el índice de ovoposición (IO), el porcentaje de inhibición de la ovoposición (IO\%), el control de reproducción (CR\%) y la eficiencia reproductiva (ER) fue determinado para cada tratamiento y controles a través de las siguientes fórmulas:

$$
\begin{aligned}
I O & =\frac{\text { Peso de los huevos }(g \mid)}{\text { Peso inicial de las hembras }(g)} \\
I O \% & =\frac{(\mathrm{IO} \text { control negativo }-\mathrm{IO} \text { extracto) }}{I O \text { control negativo }} \times 100
\end{aligned}
$$

Posteriormente, se colocaron 100 huevos de cada unidad experimental en viales de vidrio tapados con un tapón de gasa, llevándose a incubación por 21 días hasta su eclosión; determinado así el porcentaje de eclosión de larvas (E\%).

Con la IO y el E\% se calculó la ER, así: $\mathrm{ER}=\mathrm{IO} \times \mathrm{E} \%$. La ER de cada grupo de garrapatas tratado se comparó con la ER de los grupos control positivo y control negativo; luego se calculó el CR\%, así:

$C R \%=\frac{(E R \text { control negativo }-E R \text { del tratado })}{E R \text { Control negativo }} \times 100$

Para la prueba LIT se usaron larvas de 7 a 14 días de edad; exponiéndolas a diferentes concentraciones de los extractos 20,40, 80, 160 $\mathrm{mg} / \mathrm{mL}$, controles negativo y positivo igual a los descritos anteriormente; desarrollando tres repeticiones por tratamiento. Para el bioensayo los viales con larvas se colocaron en el centro de una placa de Petri que se llenó posteriormente con agua y jabón; se utilizó la metodología descrita por Klafke et al (10) donde $1 \mathrm{~mL}$ de cada solución final de inmersión se distribuyó en tres tubos plásticos para microcentrífuga de
$1.5 \mathrm{~mL}$; con el uso de un pincel de nylon $\mathrm{N}^{\circ} 4$, aproximadamente 300 larvas se transfirieron a cada tubo, el cual fue cerrado y agitado vigorosamente hasta asegurar la inmersión por 10 minutos de las larvas; posteriormente 100 larvas son retiradas y trasladadas a un papel filtro Whatman \#1 que se dobló y cerró, estos montajes fueron colocados en cajas plásticas para su incubación por 24 horas.

Se consideraron las larvas muertas: las que no se movieron, las que presentaron ataxia - movimientos apendiculares únicamente al examen estereoscópico. Para calcular la mortalidad se utilizó la fórmula:

$\%$ Mortalidad $=\frac{\text { Número de larvas muertas }}{\text { Larvas totales }} \times 100$

Pruebas in situ. La actividad acaricida de Eme y EMc se evaluó en bovinos del Hato lechero Barcelona, ubicado en el $\mathrm{Km} 12$ vía Puerto López en el municipio de Villavicencio, Meta; la evaluación del EGs se realizó en el Centro Agroindustrial del Meta "Hachón" (SENA), ubicado en el Kilómetro 17 vía Villavicencio Puerto López. El uso de animales fue con previo consentimiento informado de los propietarios y la aprobación por parte del Comité de Ética de la Universidad de los Llanos.

Para cada bioensayo se utilizaron 9 bovinos mestizos con infestación alta a media de Rm (aproximadamente 20 teleologías por animal), con edades promedio de 18 meses y peso vivo promedio de $300 \mathrm{Kg}$; los bovinos no recibieron ningún tipo de medicación acaricida por un periodo de dos meses antes del experimento; los animales pastorearon una pradera de gramíneas y tuvieron agua a voluntad; los cuales se distribuyeron en tres grupos experimentales, donde 3 animales se sometieron a un baño por aspersión con la solución de los extractos a la $\mathrm{CL}_{50} ; 3$ animales fueron el control negativo (aguaTween $802.5 \%$ ); y finalmente 3 animales como control positivo asperjados con Amitraz 0.025\%, para cada experimento. Los tratamientos en los bovinos se realizaron con bomba manual de espalda con presión, cada animal recibió tratamiento durante diez minutos con 1 litro de solución por cada $100 \mathrm{Kg}$ de peso vivo.

Para la evaluación del efecto de los extractos en los bovinos se realizó la metodología descrita por Bianchi et al (3), en la cual teleoginas (garrapatas $>4.5$ y $<8 \mathrm{~mm}$ de longitud corporal) y larvas (garrapatas $<4.5 \mathrm{~mm}$ de longitud corporal) fueron contadas del lado derecho del cuerpo del bovino, específicamente en 4 áreas de $10 \mathrm{~cm}^{2}$ : aspecto ventrolateral y dorsolateral cervical, base de la cola y periné (Figura 1 ). 


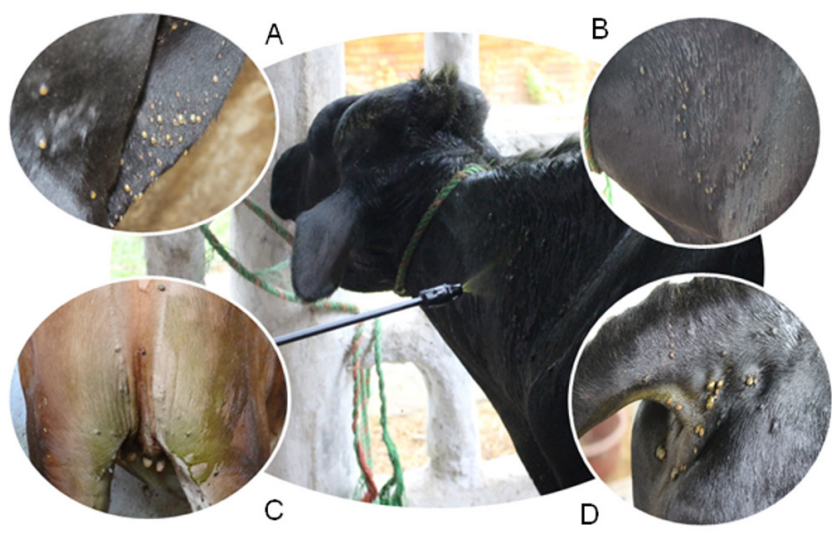

Figura 1. Evaluación dela infestación de garrapatas y conteo de estadios larvarios y teleoginas de Rm. A. Área ventrolateral cervical, B. Área dorsolateral cervical, C. Área periné, D. Área base de la cola.

El conteo de garrapatas se hizo antes del tratamiento (Día 0), días 1 (D1), día 2 (D2) y día 3 postratamiento (D3). Conteos diarios de garrapatas se efectuaron siempre a la misma hora (07:00-8 am) y por el mismo investigador. Al final del D3 se colectaron las teleoginas trasladándolas a condiciones in vitro, de tal forma se organizaron grupos de 10 teleoginas para los diferentes tratamientos, los cuales fueron llevados a incubación de acuerdo a la metodología AIT para evaluar la capacidad reproductiva después de haber recibido los baños de aspersión.

Análisis estadístico. El tamaño de la muestra de los experimentos in situ se calculó a partir de un 80 de potencia, intervalo de confianza del $95 \%$, 5\% de error máximo, promedio de $12 \pm 2$ hembras regurgitadas, a través del programa OPenEpi, versión 3.01. Los resultados de mortalidad de larvas y teleoginas fueron sometidos a la prueba de homogeneidad de varianza, prueba de Bartlett, y prueba de normalidad Kolmogorov-Smirnov, posterior análisis paramétrico a través del método Probit para el cálculo de dosis letales 50 y 90; además de Chi-cuadrado para evidenciar diferencias entre tratamientos.

Otros resultados obtenidos de las pruebas invitro (\%IO, ER, CR\%); además de los resultados obtenidos en las pruebas in-situ se sometieron a las pruebas de homogeneidad de varianza y normalidad descritas con anterioridad, para ser procesados a través del análisis de varianza de una vía y comparación múltiple de promedios con la prueba Tukey-Kramer, con un rango de confiabilidad del 95\% $(p<0.05)$. Los datos fueron organizados y analizados en el programa estadístico OpenStat 4.0, versión 7.0.

\section{RESULTADOS}

La marcha fitoquímica preliminar del EMc determinó la presencia de alcaloides, saponinas y esteroles; del EMe se obtuvo alcaloides y saponinas; y del EGs se hallaron flavonoides, terpenoides, cumarinas, glucósidos cardiotónicos, saponinas y taninos.

Pruebas in vitro. Los EMc y EMe no mostraron actividad larvicida; mientras que EGs mostró una $\mathrm{CL}_{50} 78 \mathrm{mg} / \mathrm{mL}$ (IC 71-83 mg/mL) y $\mathrm{CL}_{90}$ $146 \mathrm{mg} / \mathrm{mL}$ (IC $128-182 \mathrm{mg} / \mathrm{mL}$ ) en LIT. LoS resultados de la prueba AIT con los extractos evaluados se halló una $\mathrm{CL}_{50} 125 \mathrm{mg} / \mathrm{mL}$ (IC 69-266 $\mathrm{mg} / \mathrm{mL}$ ) y una $\mathrm{CL}_{90} 930 \mathrm{mg} / \mathrm{mL}$ (IC

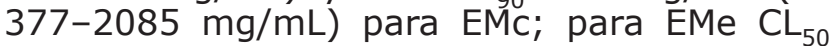
$69 \mathrm{mg} / \mathrm{mL}$ (IC 20-176 mg/mL) y CL $\mathrm{CL}_{90} 846 \mathrm{mg} /$ $\mathrm{mL}$ (IC 268-94906 $\mathrm{mg} / \mathrm{mL}$ ); y para EGs $\mathrm{CL}_{50}$ $155 \mathrm{mg} / \mathrm{mL}$ (IC 110-241 mg/mL) y una $\mathrm{CL}_{90}$ $562 \mathrm{mg} / \mathrm{mL}$ (IC 324-2601 mg/mL). Además se estableció que a la concentración de 160 mg/ $\mathrm{mL}$ evaluada sobre teleoginas, existe efectos deletéreos significativos $(p<0.05)$ sobre el ciclo de vida de este parásito (Tabla 1).

Tabla 1. Eficacia de los tres extractos vegetales in-vitro mediante la prueba AIT en la inhibición de la ovoposición y reducción de la eclosión de Rm.

\begin{tabular}{ccccc}
\hline $\mathbf{1 6 0 m g / m L}$ extracto & IO & IO\% & ER & CR\% \\
\hline Gs & 0.22 & 46.9 & 16.46 & 62.35 \\
MC & 0.164 & 66.1 & 16.40 & 66.15 \\
Me & 0.06 & 84.03 & 4.03 & 91.3 \\
\hline
\end{tabular}

Pruebas in situ. Los resultados mostrados en promedios y desviación estándar de los conteos de teleoginas del lado derecho de los animales, se evidencian en las tablas 2, 3 y 4.

Tabla 2. Efecto Mc sobre los promedios de conteos de teleoginas del lado derecho de los bovinos.

\begin{tabular}{|c|c|c|c|c|c|}
\hline ratamientos & Día 0 & Día 1 & Día 2 & Día 3 & Average \\
\hline M & $\begin{array}{c}9.33 \\
\pm 7.57^{\mathrm{A}}\end{array}$ & $\begin{array}{c}7.33 \\
\pm 5.51^{\mathrm{A}}\end{array}$ & $\begin{array}{c}6.67 \\
\pm 5.69 \mathrm{~A}\end{array}$ & $\begin{array}{c}3.67 \\
\pm 4,04\end{array}$ & $\begin{array}{c}5.89 \\
\pm 1.95^{\mathrm{B}}\end{array}$ \\
\hline $\begin{array}{l}\text { Con } \\
\text { neg }\end{array}$ & $\begin{array}{c}13.33 \\
\pm 9.02^{\mathrm{A}}\end{array}$ & $\begin{array}{c}9.33 \\
\pm 5.69 \mathrm{~A}\end{array}$ & $\begin{array}{c}11.67 \\
\pm 7.51^{\mathrm{A}}\end{array}$ & $\mathrm{N}$ & $\begin{array}{c}10.50^{ \pm} \\
1.64^{\mathrm{A}}\end{array}$ \\
\hline $\begin{array}{l}\text { Control } \\
\text { positivo }\end{array}$ & $\begin{array}{l}3.67 \\
\pm 2.31^{\mathrm{B}}\end{array}$ & $\begin{array}{c}2.67 \\
\pm 1.53^{B}\end{array}$ & $\begin{array}{c}6.67 \\
\pm 4.93^{A}\end{array}$ & $\mathrm{~N}$ & $\begin{array}{c}4.67 \\
\pm 2.82^{\mathrm{B}}\end{array}$ \\
\hline
\end{tabular}

Letras diferentes en la misma columna indican diferencias significativas $p<0.05$, ANOVA posthoc Tukey-Kramer.

Tabla 3. Efecto Me sobre los promedios de conteos de teleoginas del lado derecho de los bovinos.

\begin{tabular}{|c|c|c|c|c|c|}
\hline Tratamientos & Día 0 & Día 1 & Día 2 & Día 3 & Average \\
\hline Me & $\begin{array}{c}21.3 \\
\pm 2.3^{\mathrm{A}}\end{array}$ & $\begin{array}{c}9 \\
\pm 4^{\mathrm{A}}\end{array}$ & $\begin{array}{c}8.3 \\
\pm 2.5^{\mathrm{A}}\end{array}$ & $\begin{array}{c}9.6 \\
\pm 3.2^{\mathrm{A}}\end{array}$ & $\begin{array}{c}9 \\
\pm 0.6^{A}\end{array}$ \\
\hline $\begin{array}{c}\text { Control } \\
\text { negativo }\end{array}$ & $\begin{array}{c}4.6 \\
\pm 2.0^{\mathrm{B}}\end{array}$ & $\begin{array}{c}3.6 \\
\pm 1.5^{\mathrm{AB}}\end{array}$ & $\begin{array}{c}18.6 \\
\pm 8^{\mathrm{A}}\end{array}$ & $\begin{array}{c}32.6 \\
\pm 13.5^{\mathrm{B}}\end{array}$ & $\begin{array}{c}18.3 \\
\pm 14.5^{B}\end{array}$ \\
\hline $\begin{array}{l}\text { Control } \\
\text { positivo }\end{array}$ & $\begin{array}{c}6.6 \\
\pm 1.5^{\mathrm{B}} \\
\end{array}$ & $\begin{array}{c}1.6 \\
\pm 1.1^{\mathrm{B}} \\
\end{array}$ & $\begin{array}{c}1.3 \\
\pm 0.5^{\mathrm{B}} \\
\end{array}$ & $\begin{array}{c}0.6 \\
\pm 1.1^{\mathrm{C}} \\
\end{array}$ & $\begin{array}{c}1.2 \\
\pm 0.5^{c} \\
\end{array}$ \\
\hline
\end{tabular}

Letras diferentes en la misma columna indican diferencias significativas $p<0.05$, ANOVA posthoc Tukey-Kramer. 
Tabla 4. Efecto de Gs sobre los promedios de conteos de teleoginas del lado derecho de los bovinos.

\begin{tabular}{cccccc}
\hline Tratamientos & Día O & Día 1 & Día 2 & Día 3 & Average \\
\hline \multirow{2}{*}{$G s$} & 25 & 27 & 36 & 32.3 & 31.7 \\
& $\pm 9.54^{\mathrm{A}}$ & $\pm 9.85^{\mathrm{A}}$ & $\pm 20.52^{\mathrm{A}}$ & $\pm 18.8^{\mathrm{A}}$ & $\pm 4.5^{\mathrm{A}}$ \\
Control & 23 & 24 & 33 & $24.3^{\mathrm{A}}$ & 27.11 \\
negativo & $\pm 14.18^{\mathrm{A}}$ & $\pm 18.08^{\mathrm{A}}$ & $\pm 22.87^{\mathrm{AB}} \pm 19.14^{\mathrm{AB}}$ & $\pm 5.10^{\mathrm{A}}$ \\
Control & 27.33 & 9 & 8.33 & 5.33 & 7.56 \\
positivo & $\pm 20.03^{\mathrm{A}}$ & $\pm 6.08^{\mathrm{A}}$ & $\pm 4.73^{\mathrm{B}}$ & $\pm 3.21^{\mathrm{B}}$ & $\pm 1.95^{\mathrm{B}}$ \\
\hline
\end{tabular}

Letras diferentes en la misma columna indican diferencias significativas $p<0.05$, ANOVA posthoc Tukey-Kramer.

En cuanto a los promedios y desviaciones estándar del conteo de larvas se presentan en las tablas 5,6 y 7.

Tabla 5. Resultados prueba in-situ para Mc y sus efectos sobre estadios larvarios.

\begin{tabular}{|c|c|c|c|c|c|}
\hline Tratamientos & Día 0 & Día 1 & Día 2 & Día 3 & Average \\
\hline Mc & $\begin{array}{c}6.67 \\
\pm 7.33^{\mathrm{A}}\end{array}$ & $\begin{array}{c}2.75 \\
\pm 1.76^{\mathrm{A}}\end{array}$ & $\begin{array}{c}1.67 \\
\pm 2.35^{\mathrm{A}}\end{array}$ & $\begin{array}{c}1.17 \\
\pm 0.94\end{array}$ & $\begin{array}{c}1.86 \\
\pm 0.80^{\mathrm{B}}\end{array}$ \\
\hline $\begin{array}{c}\text { Control } \\
\text { negativo }\end{array}$ & $\begin{array}{c}4.42 \\
\pm 4.38^{\mathrm{A}}\end{array}$ & $\begin{array}{c}3.92 \\
\pm 3.68^{\mathrm{A}}\end{array}$ & $\begin{array}{c}2.83 \\
\pm 2.55^{\mathrm{A}}\end{array}$ & NR & $\begin{array}{c}3.38 \pm \\
0.76^{A}\end{array}$ \\
\hline $\begin{array}{l}\text { Control } \\
\text { positivo }\end{array}$ & $\begin{array}{c}12.08 \\
\pm 11.56^{\mathrm{A}}\end{array}$ & $\begin{array}{c}4 \\
\pm 5.13^{\mathrm{A}}\end{array}$ & $\begin{array}{c}4 \\
\pm 5.10^{\mathrm{A}}\end{array}$ & NR & 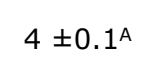 \\
\hline
\end{tabular}

Letras diferentes en la misma columna indican diferencias significativas $p<0.05$, ANOVA posthoc Tukey-Kramer.

Tabla 6. Resultados prueba in-situ para Me y sus efectos sobre estadios larvarios.

\begin{tabular}{cccccc}
\hline Tratamientos & Día 0 & Día 1 & Día 2 & Día 3 & Average \\
\hline Me & 23.7 & 15 & 19.5 & 18 & 17.5 \\
& $\pm 30.6^{\mathrm{A}}$ & $\pm 13.8^{\mathrm{A}}$ & $\pm 17.5^{\mathrm{A}}$ & $\pm 10.6^{\mathrm{A}}$ & $\pm 2.2^{\mathrm{A}}$ \\
Control & 39.1 & $20.6^{2}$ & 22.7 & 28.2 & 23.8 \\
negativo & $\pm 28^{\mathrm{A}}$ & $\pm 19.8^{\mathrm{A}}$ & $\pm 21.9^{\mathrm{A}}$ & $\pm 19.9^{\mathrm{A}}$ & $\pm 3.9^{\mathrm{B}}$ \\
Control & 17 & $11.2^{\mathrm{A}}$ & $7.3^{\mathrm{A}}$ & $7.3^{\mathrm{A}}$ & $8.6^{\mathrm{C}}$ \\
positivo & $\pm 20.8^{\mathrm{A}}$ & $\pm 8.8^{\mathrm{A}}$ & $\pm 4.5^{\mathrm{A}}$ & $\pm 4.8^{\mathrm{A}}$ & $\pm 2.2^{\mathrm{C}}$ \\
\hline
\end{tabular}

Letras diferentes en la misma columna indican diferencias significativas $p<0.05$, ANOVA posthoc Tukey-Kramer.

Tabla 7. Resultados prueba in-situ para Gs y sus efectos sobre estadios larvarios.

\begin{tabular}{cccccc}
\hline Tratamientos & Día O & Día 1 & Día 2 & Día 3 & Average \\
\hline \multirow{2}{*}{ Gs } & 15.67 & 11.67 & 11.25 & 14.17 & 12.36 \\
& $\pm 18.6^{\mathrm{A}} \pm 11.70^{\mathrm{A}} \pm 14.83^{\mathrm{A}}$ & $\pm 9.84^{\mathrm{A}}$ & $\pm 1.57^{\mathrm{A}}$ \\
Control & 11 & 12,33 & 10.33 & 14 & 12.22 \\
negativo & $\pm 13.31^{\mathrm{A}} \pm 15.29^{\mathrm{A}} \pm 13.28^{\mathrm{A}} \pm 15.96^{\mathrm{A}}$ & $\pm 1.83^{\mathrm{AB}}$ \\
Control & 21.92 & 10.67 & 8.42 & 8.42 & 9.16 \\
positivo & $\pm 20.16^{\mathrm{A}} \pm 9.63^{\mathrm{A}}$ & $\pm 7.55^{\mathrm{A}}$ & $\pm 8.15^{\mathrm{A}}$ & $\pm 1.29^{\mathrm{B}}$
\end{tabular}

Letras diferentes en la misma columna indican diferencias significativas $p<0.05$, ANOVA posthoc Tukey-Kramer.

Así mismo, en la evaluación de los efectos de los extractos vegetales sobre la capacidad reproductiva de las teleoginas recolectadas el día D3, los resultados se presentan en las tablas 8,9 y 10 .

Tabla 8. Efecto en la inhibición de la ovoposición y reducción de la eclosión de Mc sobre teleoginas colectadas D3.

\begin{tabular}{|c|c|c|c|c|}
\hline Tratamientos & IO & $10 \%$ & ER & CR\% \\
\hline Tween-80 2.5\% & $0.650^{\mathrm{A}}$ & NA & $66.25^{A}$ & NA \\
\hline Amitraz $0.025 \%$ & $0.434^{B}$ & $33.2^{\mathrm{A}}$ & $47.90^{B}$ & $33.21^{\mathrm{A}}$ \\
\hline AIT CL $\mathrm{CL}_{50} 125 \mathrm{mg} / \mathrm{mL}$ & $0.378^{\mathrm{B}}$ & $41.8^{\mathrm{A}}$ & $37.80^{B}$ & $41.83^{A}$ \\
\hline
\end{tabular}

Letras diferentes en la misma columna indican diferencias significativas $\mathrm{p}<0.05$, ANOVA posthoc Tukey-Kramer. NA, no aplica.

Tabla 9. Efecto en la inhibición de la ovoposición y reducción de la eclosión de Me sobre teleoginas colectadas D3.

\begin{tabular}{|c|c|c|c|c|}
\hline Tratamientos & IO & $10 \%$ & ER & CR\% \\
\hline Tween-80 2.5\% & $0.523^{A}$ & IVA & & NA \\
\hline Amitraz $0.025 \%$ & $0.243^{\mathrm{B}}$ & $53.5^{A}$ & $7.29^{\mathrm{B}}$ & $79.80^{\mathrm{A}}$ \\
\hline AIT $\mathrm{CL}_{50} 69 \mathrm{mg} / \mathrm{mL}$ & $0.361^{\mathrm{B}}$ & $30.9^{B}$ & $36.11^{\mathrm{C}}$ & $30.95^{B}$ \\
\hline
\end{tabular}

Letras diferentes en la misma columna indican diferencias significativas $\mathrm{p}<0.05$, ANOVA posthoc Tukey-Kramer. NA, no aplica.

Tabla 10. Efecto en la inhibición de la ovoposición y reducción de la eclosión de Gs sobre teleoginas colectadas D3.

\begin{tabular}{ccccc}
\hline Tratamientos & IO & IO\% & ER & CR\% \\
\hline Tween-80 $2.5 \%$ & $0.417^{\mathrm{A}}$ & $\mathrm{NA}$ & $41.67^{\mathrm{A}}$ & $\mathrm{NA}$ \\
Amitraz 0,025\% & $0.414^{\mathrm{A}}$ & $0.6^{\mathrm{A}}$ & $20.71^{\mathrm{B}}$ & $33.91^{\mathrm{A}}$ \\
Extracto $100 \mathrm{mg} / \mathrm{mL}$ & $0.313^{\mathrm{A}}$ & $24.8^{\mathrm{B}}$ & $31.34^{\mathrm{B}}$ & $24.78^{\mathrm{A}}$ \\
\hline
\end{tabular}

Letras diferentes en la misma columna indican diferencias significativas $\mathrm{p}<0.05$, ANOVA posthoc Tukey-Kramer. NA, no aplica.

\section{DISCUSIÓN}

No se han realizado estudios previos sobre las propiedades acaricidas de los EMc, EMe y EGs sobre las garrapatas Rm, siendo este el primer reporte. La aplicación de plantas o sus extractos para controlar microorganismos ha sido utilizada por los practicantes de la medicina tradicional; para las últimas décadas las propiedades acaricidas e insecticidas de los extractos de plantas se han usado ampliamente como control biológico de plagas (11).

La planta $M c$ ha sido ampliamente utilizada como planta medicinal, sus propiedades medicinales incluyen 
su potencial antidiabético, antiinflamatorio, antipirético, actividad antimicrobiana, antiviral, antiparasítica, antihelmíntica, antimalárica y antifúngica (12). Gs es una leguminosa arbórea usada principalmente como especie forrajera en bovinos, sus hojas tienen un alto valor nutricional con proteína cruda $23 \%$ (13); esta planta se utiliza como un antihistamínico, antipirético, expectorante, diurético, antioxidante, antimicrobiana, antimalárica, antihelmíntica, tripanocida, mosquicida y efecto acaricida $(14,15)$.

Por otra parte Me es un arbusto de floración muy vistosa y utilizada comúnmente como planta de ornamentación (16), a diferencia de las otras plantas esta no reporta información acerca de otros usos posibles.

Diversos estudios han demostrado la capacidad de grupos de metabolitos secundarios tipo cumarinas, taninos condensados, flavonoides, triterpenos, rotenoides, entre otros; para alterar la biología de ácaros e insectos $(15,17,18,19)$. Varios de estos grupos de metabolitos secundarios fueron encontrados en la marcha fitoquímica de EGs, EMc y EMe colectadas en condiciones del piedemonte del Meta. A partir de la determinación de estos grupos químicos promisorios desde la perspectiva antiparasitaria externa e interna se han desarrollado varios estudios; por ejemplo en pruebas realizadas con extractos de Gs se observó su efecto antihelmíntico invitro reduciendo la motilidad de larvas estadio $\mathrm{L}_{3}$ de Haemonchus contortus; este efecto se relacionó con metabolitos secundarios tales como taninos/compuestos polifenólicos (20), también a terpenoides, flavonoides y lectinas (21); así mismo se ha demostrado que plantas ricas en estos mismos grupos de metabolitos secundarios han mostrado actividad acaricida sobre $\operatorname{Rm}(22,23,24,25)$.

Por otro lado, en el grupo de metabolitos secundarios tipo cumarinas, grupo determinado en el EGs, se ha asociado su capacidad antiparasitaria sobre ácaros a través de la inhibición de enzimas digestivas que pertenecen a la familia de las endopeptidasas tipo serina, cisteina, aspartato; además metaloproteasas, tripsina, quimotripsina y catepsinas (26), enzimas con capacidad proteolítica de $\mathrm{Rm}$ quienes son las encargadas de la digestión de la hemoglobina y actúan como proteínas de reserva durante la embriogénesis (27). Posiblemente este mecanismo podría explicar los resultados obtenidos en la potente inhibición del ciclo de vida de Rm (\%IO, CR\%, ER; Tablas 1, 8-10) al afectar los estadios larvarios y adultos utilizados en el estudio; y las diferencias significativas sobre la mortalidad en los estadios larvarios y teleoginas (Tablas 2-7); siendo estas enzimas vitales en la supervivencia de este ácaro.
Algunos estudios han evidenciado la actividad acaricida in vitro de Gs sobre Rm, con resultados similares a los encontrados en esta investigación (Tabla 1), por ejemplo Rodríguez et al (28) evaluaron la eficacia in vitro del extracto de Morus alba y Gs en el control Rm y su oviposición; en el cual el extracto puro de Gs manifestó una mortalidad del $53.33 \%$; así mismo obtuvieron una IO del $58 \%$.

Aunque la actividad acaricida in vitro sobre teleoginas de $\mathrm{Rm}$ expuestos a los extractos vegetales de este estudio muestran resultados promisorios de la incursión de estas plantas dentro del control integrado de parásitos externos en sistemas de producción bovina del piedemonte del departamento del Meta (Tabla $1,2,3,4,5,6,7)$.

El interés de este tipo de fitoterapéutico recae sobre la inviabilidad de los huevos de este parasito; encontrándose para este estudio resultados significativos al obtener un $62.35 \%$, $66.15 \%$ y $91.3 \%$ de CR\% y una ER\% del 16.46 , $16.40 \%$ y $4.03 \%$, para Gs, Mc y Me; dentro del ciclo de vida de la $\mathrm{Rm}$ a la concentración del $16 \%$ de los extractos (Tabla 1), es decir, los extractos bajo este modelo de experimentación generan un fenómeno de inhibición en el proceso de eclosión (CR\%) por encima del 50\% de los huevos ovopositados por las teleoginas expuestas a estos fitopreparados.

Datos similares fueron reportados por Álvarez et al (15) donde el extracto alcohólico de Gs mostró un efecto signifcativo $(p<0.05)$ sobre el $\mathrm{IO} \%$ en $\mathrm{Rm}$ del $94 \%$. Así mismo sobre su capacidad acaricida se ha publicado que el extracto etanólico $5 \%$ de hojas de Gs redujo un $57 \%$ la ovoposición del acaro Tetranychus cinnabarinus, obtenido de plantas de frijol negro; además, concentraciones de 5, 10, 15, y $20 \%$ del extracto etanólico de la planta causaron $41.7,80.6,88.9$ y $100 \%$ de mortalidad, respectivamente (14).

Por estas razones los resultados obtenidos del estudio in-vitro e in-situ desarrollado validan los reportes etnofarmacológicos del uso de las plantas Gs y Mc $(29,30)$ como acaricidas; además de vincular como nueva candidata a la planta Me como fitoterapéuticos promisorios en sistemas de producción animal.

Por otra lado, en los hatos bovinos del trópico bajo la carga parasitaria de la población se establece en un 92\% asociada al agroecosistema (fases preparasíticas o de vida libre) y tan sólo un $8 \%$ o menos está en su estadio parasitario obligado; resaltando la importancia de vincular dentro del control integrado de parásitos la oportunidad de controlar las fases preparasíticas del agente 
parasitario a tratar; oportunidad que debe estar soportada en procesos y procedimientos sustentables desde la perspectiva ambiental (11).

En ese momento del control parasitario los extractos vegetales jugarian un importante papel, al poder ser utilizados en la disminución de la carga parasitaria libre con mínimo impacto medio ambiental (30); además de su posible uso sobre el bovino, acción que debe ser validada a través de futuros estudios in-vivo e in-situ que permitan comparar los resultados obtenidos en este estudio. La anterior información es relevante y puede ser usada para consolidar el cultivo del Gs, Mc y Me en los sistemas de producción bovino del piedemonte del departamento del Meta, con la finalidad de ser utilizada como método alternativo dentro del control integrado de la garrapata común del ganado, fortaleciendo el desarrollo silvopastoril de la ganadería colombiana en el marco de la apertura económica del reglón productivo primario o extractivo (1).

\section{Conflicto de intereses}

Los autores declararón que no existen conflictos de interés potenciales con respecto a la investigación, autoría o publicación de este artículo.

\section{Agradecimientos}

Al Fondo Nacional de Financiamiento para la Ciencia, la Tecnología y la Innovación, Francisco José de Caldas y la Universidad de los Llanos; por la financiación de este trabajo dentro del Proyecto de investigación: "Alternativa terapéutica para el control de Rhipicephalus microplus en bovinos con base en plantas forrajeras del piedemonte del Meta" Código 1122-569-35063; y al profesor Carlos Enrique Hoyos Diez, de la Facultad de Ciencias Humanas y de la Educación, por la traducción del manuscrito.

\section{REFERENCIAS}

1. CONPES. Documento CONPES 3676: Consolidación de la política sanitaria y de inocuidad para las cadenas láctea y cárnica. Colombia, Bogotá: Departamento Nacional de Planeación, Consejo Nacional de Política Económica y Social, Departamento Nacional de Planeación. 2010. URL Available from: https://colaboracion.dnp.gov.co/CDT/ Conpes/Econ\%C3\%B3micos/3676.pdf

2. Reck J, Berger M, Terra RM, Marks FS, da Silva I, Guimarães JA, Termignoni C. Systemic alterations of bovine hemostasis due to Rhipicephalus (Boophilus) microplus infestation. Res Vet Sci. 2009; 86(1):56-62. [CrossRef] [PubMed] [Google Scholar]

3. Bianchi MV, Barré N, Messad S. Factors related to cattle infestation level and resistance to acaricides in Boophilus microplus tick populations in New Caledonia. Vet Parasitol. 2003; 112(1-2):75-89. [CrossRef] [PubMed] [Google Scholar]
4. FAO. Maximum residue limits (MRLs) and risk management recommendations (RMRs) for residues of veterinary drugs in foods. FAO/WHO Food Standards Programme. Food and Agriculture Organization of the United Nations. 2017. URL Available from: http:// www.fao.org/fao-who-codexalimentarius/ codex-texts/maximum-residue-limits/en/

5. Montes-Molina JA, Luna-Guidoa ML, Espinoza-Paz N, Govaertsc B, GutierrezMicelid FA, Dendooven L. Are extracts of neem (Azadirachta indica A. Juss. (L.)) and Gliricidia sepium (Jacquin) an alternative to control pests on maize (Zea mays L.)? Crop Prot. 2008; 27(3-5):763-774. [CrossRef] [Google Scholar]

6. Castelblanco L, Sanabria OJ, Cruz A, Rodríguez CE. Reporte preliminar del efecto ixodicida de extractos de algunas plantas sobre garrapatas Boophilus microplus. Rev Cubana Plant Med. 2013; 18(1):118-130. [Google Scholar] 
7. García-Barriga H. Flora Medicinal de Colombia. Tomos I y II. Bogotá, Colombia: Imprenta Nacional de Colombia; 1975. [Google Scholar]

8. Sanabria A. Análisis fitoquímico preliminar. Metodología y su aplicación en la evaluación de 40 plantas de la familia Compositae. Bogotá, Colombia: Universidad Nacional de Colombia, Facultad de Ciencias, Departamento de Farmacia. 1983. https:// books.google.com.co/books/about/ Analisis fitoquimico preliminar metodolo. html?id =i2vhMgEACAAJ\&redir esc $=y$

9. Drummond RO, Glandey WJ, Whetstone T, Ernest SE. Laboratory testing of insecticides for control of the winter tick. J Econ Entomol. 1971; 64(3):686-688. [CrossRef] [PubMed] [Google Scholar]

10. Klafke GM, Castro-Janer E, Mendes MC, Namindome A, Schumaker TT. Applicability of in vitro bioassays for the diagnosis of ivermectin resistance in Rhipicephalus microplus (Acari: Ixodidae). Vet Parasitol. 2012; 184(2-4):212-220.[CrossRef] [PubMed] [Google Scholar]

11. FAO. Animal Production and Health Division. Resistance Management and Integrated Parasite Control in Ruminants. Italy, Rome: Animal Production and Health Division, Agriculture Dept., Food and Agriculture Organization of the United Nations. 2004. URL Available from: http://www.fao.org/tempref/ docrep/fao/010/ag014e/ag014e00.pdf

12. Dvorkin-Camiel L, Pharm DJS, Whelan MS. Tropical American Plants in the Treatment of Infectious Diseases. J Diet Suppl. 2008; 5(4):349-372. [CrossRef] [PubMed] [Google Scholar]

13. Cuervo JA, Narváez SW, Hahn C. Características forrajeras de la especie Gliricidia sepium (Jacq.) stend, fabaceae. Bol Cient Mus Hist Nat. 2013; 17(1):33-45. [Google Scholar]

14. Sivira $A$, Sanabria $M E$, Valera $N$, Vásquez C. Toxicity of ethanolic extracts from Lippia origanoides and Gliricidia sepium to Tetranychus cinnabarinus (Boisduval) (Acari: Tetranychidae). Neotrop Entomol. 2011; 40(3):375-379. [CrossRef] [PubMed] [Google Scholar]
15. Álvarez V, Loaiza J, Bonilla R, Barrios M. Control in vitro tick (Boophilus microplus ; Acari: Ixodidae) through plant extracts. Rev Biol Trop. 2008; 56(1):291-302. [CrossRef] [PubMed] [Google Scholar]

16. Bufford J, Lurie M, Daehler C. Biotic resistance to tropical ornamental invasion. Journal of Ecology. 2016, 104:518-530. [CrossRef] [Google Scholar]

17. Joji RI, Beena, J. Chemical composition and antibacterial activity of the volatile oil from the bark of Gliricidia sepium. Int J Pharmacy Pharm Sci 2010; 2(3):177-179. https://innovareacademics.in/journal/ijpps/ Vol2Issue3/651.pdf

18. Krishnappa K, Dhanasekaran S, Elumalai K. Larvicidal, ovicidal and pupicidal activities of Gliricidia sepium (Jacq.) (Leguminosae) against the malarial vector, Anopheles stephensi Liston (Culicidae: Diptera). Asian Pac J Trop Med 2012; 5(8):598-604. [CrossRef] [PubMed] [Google Scholar]

19. Thomas, J., Govindan, S., Kurup, M. (2014). Isolation and characterization of mosquito larvicidal compound from Gliricidia sepium Jacq. Int J Pharm Res Health Sci 2(2):173178. http://www.pharmahealthsciences. net/pdfs/volume2/13 MS 1443.pdf

20. von Son-de Fernex E, Alonso-Dayz MA, Valles-de la Mora B, Capetillo-Leal CM. In vitro anthelmintic activity of five tropical legumes on the exsheathment and motility of Haemonchus contortus infective larvae. Exp Parasitol 2012; 131(4):413-8. [CrossRef] [PubMed] [Google Scholar]

21. Puerto $M$, Arece J, López $Y$, Roche $Y$, Molina $M$, Sanavria A, da Fonseca HA. Efecto in vitro de extracts acuosos de Moringa oleifera y Gliricida sepium en el desarrollo de las fases exógenas de estrongílidos gastrointestinales de ovinos. Rev Salud Anim. 2014; 36(1):2834. http://scielo.sld.cu/scielo.php?script $=$ sci arttext\&pid=S0253-570X2014000100005

22. Bagavan A, Kamara JC, Elango G, Zahir $A A$, Rahuman AA. Adulticidal and larvicidal efficacy of some medicinal plant extracts against tick, fluke and mosquitoes. Vet Parasitol. 2009; 166(3-4):286-292. [CrossRef] [PubMed] [Google Scholar] 
23. Zorloni A, Penzhorn BL, Eloff JN. Extracts of Calpurnia aurea leaves from southern Ethiopia attract and immobilise or kill ticks. Vet Parasitol. 2010; 168(1-2):160-164. [CrossRef] [PubMed] [Google Scholar]

24. Dantas ACS, Machado DMR, Araujo AC, Oliveira-Junior RG, Lima-Saraiva SRG, Ribeiro LAA, Almeida JRGS, Horta MC. Acaricidal activity of extracts from the leaves and aerial parts of Neoglaziovia variegata (Bromeliaceae) on the cattle tick Rhipicephalus (Boophilus) microplus. Res Vet Sci 2015; 100:165-168. https://doi. org/10.1016/j.rvsc. 2015.04.012 [CrossRef] [PubMed] [Google Scholar]

25. Ghosh S, Shankar S, Srivastava S, kumar S, Kumar A, Nagar G. et al. In vitro acaricidal properties of Semecarpus anacardium fruit and Datura stramonium leaf extracts against acaricide susceptible (IVRI-I line) and resistant (IVRI-V line) Rhipicephalus (Boophilus) microplus. Res Vet Sci 2015; 101:69-74. [CrossRef] [PubMed] [Google Scholar]

26. Estrela $A B$, Seixas $A$, Teixeira $O$, Pinto $A F$, Termignoni $C$. Vitellin- and hemoglobindigesting enzymes in Rhipicephalus (Boophilus) microplus larvae and females. Comp Biochem Physiol B Biochem Mol Biol 2010; 157(4):326-335. [CrossRef] [PubMed] [Google Scholar]
27. Fagotto F. Yolk degradation in tick eggs: I Ocurrence of Cathepsin L-like acid proteinase in yolk spheres. Arch Insect Biochem Physiol 1990; 14(4):217-235. [CrossRef] [PubMed] [Google Scholar]

28. Rodríguez MCE, Pulido SNJ. Eficacia de extracts vegetales sobre la garrapata adulta Rhipicephalus (Boophilus) microplus y su oviposición. Rev Cubana Plant Med 2015; 20(4):375-388. http://www. revplantasmedicinales.sld.cu/index.php/ $\mathrm{pla/article/view/230}$ [Google Scholar]

29. Brandão $M G L$, Zanetti NNS, Oliveira $P$, Grael CFF, Santos ACP, Monte-Mór RL. Brazilian medicinal plants described by 19 th century European naturalists in the Official Pharmacopoeia. J Ethnopharmacol 2008; 120(2):141-148. [CrossRef] [PubMed] [Google Scholar]

30. Ribeiro VL, Avancini C, Gonçalves K, Toigo E, von Poser G. Acaricidal activity of Calea serrata (Asteraceae) on Boophilus microplus and Rhipicephalus sanguineus. Vet Parasitol2008; 151(2-4):351-354. [CrossRef] [PubMed] [Google Scholar] 